\title{
Variabilidade espacial de propriedades químicas do solo e da produtividade de citros na Amazônia Oriental
}

\author{
Paulo C. G. Oliveira ${ }^{1}$, Paulo R. S. Farias ${ }^{2}$, Herdjania V. Lima ${ }^{2}$, Antonio R. Fernandes ${ }^{2}$, Francisco A. Oliveira ${ }^{2}$ \& Javier D. Pita ${ }^{2}$
}

\begin{abstract}
RESUMO
Propôs-se, com este trabalho, avaliar a variabilidade espacial da concentração de macronutrientes em laranjeiras e no solo, correlacionando-as com a produtividade e o tamanho dos frutos. O estudo foi realizado em um pomar de laranjas "Pêra-Rio" implantado em um Argissolo Amarelo de textura média, localizado no município de Capitão Poço, PA. Para a análise foliar selecionaram-se 120 plantas e se coletaram as $3^{\mathrm{a}}$ e $4^{\mathrm{a}}$ folhas dos ramos, no perímetro médio da altura da copa, abrangendo todos os quadrantes, totalizando 50 folhas por planta. A coleta de solo foi realizada na camada de 0-20 cm de profundidade, considerando-se a projeção da copa em todos os quadrantes. Os dados foram submetidos a análises de geoestatística. Através dos mapas de krigagem foi possível determinar as áreas com alta e baixa variabilidade, o que permitiu concluir que os mapas de produtividade e de tamanho de fruto mostraram alta variabilidade espacial,

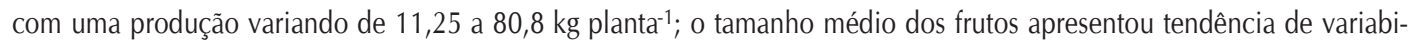
lidade, seguindo as linhas de plantio, causado, provavelmente, pelo sistema de alternância de capinas; o tamanho dos frutos variou de 42 a $78 \mathrm{~mm}$ e a produtividade não foi influenciada pelo tamanho do fruto e, sim, pelo número de frutos por planta.
\end{abstract}

Palavras-chave: geoestatística, macronutrientes, agricultura de precisão

\section{Spatial variability of soil chemical properties and yield of citrus orchards in Eastern Amazonia}

\begin{abstract}
The spatial variability of macronutrient content was analyzed in an orange orchard (cv. "Pera Rio"), set up in a typic Hapludalf soil. The correlation between yield and fruit size was determined. The experimental area was located at Capitão Poço, in the eastern part of Amazonia region, Pará State. Leaf analysis was performed in 120 plants and the leaves of third and fouth branches were collected in all quadrants, totalizing 50 leaf samples for each plant. The soil was sampled at $0-20 \mathrm{~cm}$ depth below the crop canopy, in accordance to leaf sampling location. Leaf and soil data variability were measured with geoestatistics technique and the orchard areas of higher and lower variability were assessed. The yield maps and fruit size showed high spatial variability. The total fruit production ranged from 11.25 to $80.8 \mathrm{~kg} \mathrm{plant}^{-1}$, the average fruit size varied with the plant row, probably because of hoeing in alternate plant rows. The fruit size varied from 42 to $78 \mathrm{~mm}$. The orange yield was affected only by the number of fruits per tree.
\end{abstract}

Key words: geostatistics, macronutrients, precision agriculture

1 CEFET, Centro de Educação Tecnológica do Estado do Pará, Av. Almirante Barroso, 1155 - Marco, CEP 66093-020. Belém, PA. Fone: (91) $3210-5242$. E-mail: pcbenfica@yahoo.com.br

${ }^{2}$ Instituto de Ciências Agrárias/UFRA, Av. Presidente Tancredo Neves 2501, Montese, CEP 66077-530, CP 917, Belém, PA. E-mail: paulo.farias@ufra.edu.br; herdjania.lima@ufra.edu.br; antonio.fernandes@ufra.edu.br; francisco.oliveira@ufra.edu.br 


\section{INTRODUÇÃO}

Em escala mundial, a citricultura alcança aproximadamente 108,5 milhões t/ano, sendo cultivada em 7,3 milhões de hectares superando, em grande parte, outras frutíferas tropicais e subtropicais, como banana, maçã, manga, pêra, pêssego e mamão. O Brasil e os Estados Unidos são os maiores produtores de laranja do mundo e, juntos, representam cerca de $47,7 \%$ do total produzido sobressaindo-se deste panorama o México, a Índia e a China (FAO, 2005).

Com mais de 1 milhão de hectares de plantas cítricas em seu território, o Brasil se tornou, na década de 1980, o maior produtor mundial. A maior parte da produção brasileira de laranjas se destina à indústria do suco, concentrada no estado de São Paulo, responsável por 85\% das laranjas e por 98\% do suco concentrado que o Brasil produz; no entanto, a produtividade nos EUA é superior e esta variável deve ser melhorada nos pomares brasileiros.

No início da década de 1990 o estado do Pará, devido as suas condições edafoclimáticas serem satisfatórias para esta cultura, entrou no cenário dos produtores de citros e hoje já é o quinto maior produtor do Brasil (IBGE, 2005), em particular os municípios de Capitão Poço, Irituia, Garrafão do Norte e Ourém; integrantes da microrregião do Guamá, se destacam como os maiores produtores do estado do Pará. O pólo citrícola do Estado do Pará se encontra em uma região cujos solos são de baixa fertilidade natural, ácidos e com baixa saturação por bases e, aliada a este fato, a quase ausência de tratamentos fitossanitários, razão pela qual são utilizadas elevadas doses de fertilizantes que podem causar danos ao meio ambiente e têm elevados os custos de produção.

O conhecimento das concentrações químicas dos nutrientes no solo, associado à análise do tecido vegetal (folhas) é de fundamental importância para qualquer tipo de cultura, pois é fator primordial para a caracterização da fertilidade do solo e do estado nutricional das plantas, que são fatores determinantes da produtividade, mas a avaliação dessas características, mesmo em áreas homogêneas, apresenta variação espacial, mesmo a curtas distâncias, que pode influenciar a produtividade das culturas. Fundamentada neste princípio, a geoestatística assume grande relevância possibilitando determinar a variabilidade espacial de características do solo e da planta, o que é fundamental para o entendimento de suas inter-relações.

A variabilidade espacial poderá mostrar, ao produtor, a localização exata das áreas de maior e menor produção, a concentração dos nutrientes no solo e o teor nas plantas, tornando possível, com isto, fazer a adubação e as correções devidas nos locais corretos, diminuindo consideravelmente o custo da produção e trazendo também uma enorme contribuição ao meio ambiente introduzindo, assim, a agricultura de precisão utilizada nos centros mais desenvolvidos do País (Farias et al., 2003).

Sob a égide da utilização racional do solo, que implica, dentre outras coisas, na redução da utilização dos insumos agrícolas, no sentido de evitar impactos ambientais e dos custos de produção, tem-se como ferramenta de grande potencialidade a agricultura de precisão, o que proporciona, ao agri- cultor, o mapeamento do solo, a aplicação de insumos e das atividades de colheita, considerando-se a área de modo diferenciado, a fim de racionalizar o uso, visando a um desenvolvimento sustentável, aproximando-se do ideal, que seria manejar cada planta e local, individualmente, fato impraticável econômica e tecnicamente. Apesar do Estado do Pará ocupar lugar de destaque na produção nacional, estudos sobre a variabilidade da produção e da eficiência das técnicas de manejo (insumos, fertilizantes, pragas, doenças e compactação, dentre outras) são incipientes, de modo que a produtividade média anual vem diminuindo ao longo dos anos.

Neste trabalho se objetivou avaliar a variabilidade espacial da concentração de macronutrientes no solo e nas plantas e o potencial de produtividade em pomar de citros na região de Capitão Poço, PA.

\section{MATERIAL E MÉTODOS}

\section{Localização}

A área experimental está localizada no município de Capitão Poço, PA, microrregião do Guamá, mesorregião do nordeste paraense, na Fazenda Citropar, compreendidas nas coordenadas geográficas: $01^{\circ} 48^{\prime} 38^{\prime \prime}$ de latitude Sul, $47^{\circ} 11$ ’ $38^{\prime \prime}$ de longitude Oeste de Greenwich (sede da fazenda), apresentando solo do tipo Argissolo Amarelo, textura média, clima tipo Ami, segundo a classificação de Köppen, com os maiores índices de pluviosidade de janeiro a maio, e os menores de agosto a novembro. A área está cultivada com cerca de 1 milhão de laranjeiras, com predominância da variedade PêraRio (Citrus sinensis [L.] Osb.) enxertada em tangerineira 'Cleópatra’ (Citrus reshni hort. ex. Tanaka) plantadas em espaçamentos de $5 \mathrm{~m} \mathrm{x} 7 \mathrm{~m}$, com dez anos de idade.

Os pontos foram coletados por um receptor GPS V Plus, fabricado pela Garmin. As coordenadas utilizadas no trabalho foram transformadas para o sistema UTM (Universal Transversa de Mercator), seguindo o sistema das coordenadas retangulares; selecionou-se para a coleta dos pontos, uma quadra com 4600 plantas em uma área de 13 hectares. A digitalização dos pontos amostrais foi feita no software SUFER 8.0, o qual permitiu que se construísse uma base digital da imagem de satélite da área estudada.

Os pontos usados para construção da base da imagem foram a maior e a menor coordenada (latitude e longitude) do talhão, seguindo a metodologia de Farias et al. (2003). A quadra, denominada DQ-12, foi georreferenciada e, deste universo de laranjeiras, foram selecionadas, para a amostragem, 120 plantas, em que cada planta teve sua localização espacial conhecida e, conseqüentemente, todas as informações das amostragens com precisão e confiabilidade, sendo que no centro da área houve um adensamento maior dos pontos amostrais para estimar a variabilidade em pequena distância.

\section{Amostragem e análise laboratorial}

Após a seleção de 120 plantas se coletaram as $3^{\mathrm{a}}$ e $4^{\mathrm{a}}$ folhas dos ramos, a partir do fruto, no perímetro médio da altura da copa, abrangendo todos os quadrantes, totalizando 
50 folhas por planta. As folhas amostradas foram lavadas com água destilada e detergente a $0,1 \%$; posteriormente, fezse a lavagem com $\mathrm{HCl}$ na concentração de $3 \%$ em volume e novamente se utilizou água destilada para eliminação das impurezas; após esta lavagem, as amostras foram secadas em estufa com temperatura variando de 65 a $70^{\circ} \mathrm{C}$, até peso constante; em seguida, foram moídas em moinho do tipo Wiley e acondicionadas em sacos plásticos.

Para realização das análises químicas, o material vegetal foi digerido em ácido nítrico e perclórico concentrados, resultando em extratos que foram utilizados para a determinação de teores totais dos seguintes nutrientes: $\mathrm{P}$, por colorimetria de molibdato-vanadato; $\mathrm{K}$, espectrofotometria de chama e $\mathrm{Ca}+\mathrm{Mg}$ por espectrofotometria de absorção atômica (Silva, 1999). A determinação do N se deu através de digestão sulfúrica de 200 mg de matéria seca, com destilação em aparelho micro-kjeldahl e titulação com $\mathrm{H}_{2} \mathrm{SO}_{4}$ a $0,01 \mathrm{~N}$.

A amostragem do solo foi efetuada nas 120 plantas selecionadas para análise foliar, na profundidade de $0-20 \mathrm{~cm}$, considerando-se a abrangência da projeção da copa em todos os quadrantes, totalizando 8 amostras simples por planta para obtenção de uma amostra composta. As amostras foram secadas ao ar, em ambiente protegido de contaminações, até atingirem peso constante; posteriormente se realizaram as análises de $\mathrm{pH}\left(\mathrm{H}_{2} \mathrm{O}\right.$ e $\left.\mathrm{KCl}\right)$, P e $\mathrm{K}$ (Mehlich), $\mathrm{Ca}+\mathrm{Mg}$ (extração com $\mathrm{KCl} 1 \mathrm{M}), \mathrm{H}+\mathrm{Al}$ (acetato de cálcio em pH7) conforme EMBRAPA (1997).

\section{Análise da dependência espacial (Geoestatística)}

A partir do conhecimento preliminar dos dados estatísticos das variáveis da área em estudo, passou-se à análise variográfica, procurando-se conhecer o grau de dependência espacial dessas variáveis e as características qualitativas da regionalização. As análises variográficas foram elaboradas para uma direção global isotrópica dessas variáveis no espaço, permitindo o ajuste de modelos matemáticos a esses semivariogramas. Considerando-se que a malha de amostragem da Quadra DQ12 apresenta distribuição regular, a pesquisa de pares de pontos no espaço foi feita para a direção global, ou seja, adotando-se um ângulo de tolerância de $90^{\circ}$, isto é, que os pares (dependência espacial) foram procurados nas principais direções do espaço da grade de amostragem.

Buscando-se uma definição dos parâmetros variográficos, várias tentativas foram feitas com ajustes aos modelos esférico, exponencial e gaussiano, com diferentes distâncias entre pares. Em todos os ajustes de pares, para estimar a dependência espacial das variáveis o número de pares foi sempre maior que 30; o número de pares e as distâncias estão de acordo com Guerra (1988) que cita que o número de pares com que foi calculado o semivariograma experimental desempenha papel significativo, sendo necessário pelo menos 30 pares; quanto à distância máxima, o mesmo autor afirma que, na prática, a área de estudo variográfico raramente ultrapassa a metade do campo total amostrado.

Os procedimentos a serem descritos seguem a metodologia de Farias et al. (2002a) e se utilizam da informação da posição da amostra e o valor que as variáveis assumiram em cada ponto (planta); desta forma se tem, de cada ponto de amostragem, o valor das variáveis e as coordenadas (latitude, longitude).

\section{Análise variográfica}

Avaliou-se a dependência das amostras através da geoestatística, sendo construído um semivariograma (Eq. 1) conforme Vieira et al. (1983):

$$
\gamma(\mathrm{h})=\frac{1}{2 \mathrm{~N}(\mathrm{~h})} \sum_{\mathrm{i}=1}^{\mathrm{N}(\mathrm{h})}\left[\mathrm{Z}\left(\mathrm{x}_{\mathrm{i}}\right)-\mathrm{Z}\left(\mathrm{x}_{\mathrm{i}}+\mathrm{h}\right)\right]^{2}
$$

donde $\mathrm{N}(\mathrm{h})$ é o número de pares experimentais de valores medidos $\mathrm{Z}\left(\mathrm{x}_{\mathrm{i}}\right), \mathrm{Z}\left(\mathrm{x}_{\mathrm{i}}+\mathrm{h}\right)$, separados por um vetor $\mathrm{h}$. O gráfico de $\gamma(\mathrm{h})$ "versus" valores correspondentes de h, chamado semivariograma, é uma função do vetor h e, portanto depende da magnitude e direção de h.

Após o ajuste do semivariograma aos modelos gaussiano, esférico e exponencial, os dados das amostragens foram estimados através da krigagem ordinária. Para propriedades espacialmente dependentes, espera-se que a diferença entre valores $\left[\mathrm{Z}\left(\mathrm{x}_{\mathrm{i}}\right)-\mathrm{Z}\left(\mathrm{x}_{\mathrm{i}}+\mathrm{h}\right)\right]$ seja, em média, crescente com a distância até determinado ponto a partir do qual se estabiliza um valor, denominado patamar $\left(\mathrm{C}_{1}\right)$ que é aproximadamente igual à variância dos dados. Esta distância recebe o nome de alcance (a) e representa o raio de um círculo dentro do qual os valores são tão parecidos uns com os outros que se correlacionam. O valor da semivariância na interseção do eixo Y tem o nome de efeito pepita $\left(\mathrm{C}_{0}\right)$ e representa a variabilidade da propriedade estudada em espaçamentos menores que o amostrado; assim, quanto maior o efeito pepita mais fraca é a dependência espacial de um atributo. Os mapas foram gerados através do programa Surfer 8.0 (Golden Software, 2002), conforme descrito por Farias et al. (2002b).

\section{RESULTADOS E DISCUSSÃO}

Os resultados referentes à análise descritiva para as variáveis químicas no solo e na planta são apresentados na Tabela 1 , na qual se notou que os valores das médias são, quase sempre, superiores às variâncias, indicando uma distribuição que tende para uma dependência espacial; as exceções ocorreram para o P (40,33), no solo, e para o Ca $(142,26)$, na planta. A observação de variâncias maior que a média é comum em estudos de variabilidade espacial e mostram uma distribuição agregada (Wilson \& Room, 1983).

Observa-se, na Tabela 1, que o coeficiente de variação do K no solo $(38,89 \%)$ ultrapassou os $30 \%$, indicando presença de altos valores ou discrepante (Kim, 1988). Pelos valores máximos e mínimos, nota-se que o $\mathrm{K}$ no solo apresenta grande variabilidade, o que justifica o CV alto. Souza et al. (1997) citam resultados similares ao concluirem que o K no solo é uma das propriedades mais afetadas pelo manejo, em termos de variabilidade.

De maneira geral, todas as variáveis mostram forma assimétrica para as distribuições de freqüência. A assimetria positiva dessas variáveis ocorre em virtude de uma grande concentração de valores em um número reduzido de classes; 
Tabela 1. Estatística descritiva das análises químicas do solo e da planta

\begin{tabular}{|c|c|c|c|c|c|c|c|c|c|}
\hline Variáveis & N & $\hat{\mathbf{m}}$ & $s(\hat{m})$ & $s^{2}$ & CV\% & $\mu_{3}$ & $\mu_{4}$ & Máx & Min \\
\hline \multicolumn{10}{|c|}{ Solo } \\
\hline $\mathrm{pH}$ & 120 & 4,95 & 0,26 & 0,07 & 5,25 & 0,21 & 0,34 & 5,76 & 4,20 \\
\hline M.0. $\left(\mathrm{g} \mathrm{kg}^{-1}\right)$ & 120 & 15,03 & 1,62 & 2,62 & 10,78 & 0,39 & 0,13 & 19,46 & 11,30 \\
\hline $\mathrm{P}\left(\mathrm{mg} \mathrm{dm}^{-3}\right)$ & 120 & 25,27 & 6,35 & 40,33 & 25,13 & 0,44 & 0,13 & 47,00 & 12,40 \\
\hline $\mathrm{K}\left(\mathrm{cmolc} \mathrm{dm}^{-3}\right)$ & 120 & 0,18 & 0,07 & 0,01 & 38,89 & 5,86 & 50,49 & 0,80 & 0,10 \\
\hline $\mathrm{Ca}\left(\mathrm{cmolc} \mathrm{dm}^{-3}\right)$ & 120 & 1,25 & 0,34 & 0,12 & 27,20 & 0,49 & 1,27 & 2,41 & 0,45 \\
\hline $\mathrm{Mg}\left(\mathrm{cmol}_{\mathrm{c}} \mathrm{dm}^{-3}\right)$ & 120 & 0,43 & 0,12 & 0,14 & 27,91 & 0,61 & 0,54 & 0,67 & 0,08 \\
\hline \multicolumn{10}{|c|}{ Planta } \\
\hline$N\left(g_{k g}^{-1}\right)$ & 120 & 21,23 & 2,21 & 4,87 & 10,41 & 0,42 & 2,18 & 28,82 & 13,17 \\
\hline$P\left(g_{k g}^{-1}\right)$ & 120 & 1,36 & 0,13 & 0,018 & 9,56 & 0,78 & 1,54 & 1,84 & 1,00 \\
\hline $\mathrm{K}\left(\mathrm{g} \mathrm{kg}^{-1}\right)$ & 120 & 5,11 & 0,89 & 0,79 & 17,42 & 0,45 & 0,10 & 7,71 & 3,22 \\
\hline $\mathrm{Ca}\left(\mathrm{g} \mathrm{kg}^{-1}\right)$ & 120 & 66,91 & 11,92 & 142,26 & 17,81 & 0,25 & 0,15 & 93,23 & 32,11 \\
\hline $\operatorname{Mg}\left(\mathrm{g} \mathrm{kg}^{-1}\right)$ & 120 & 4,27 & 0,70 & 0,49 & 16,39 & 0,59 & 1,35 & 7,04 & 2,80 \\
\hline
\end{tabular}

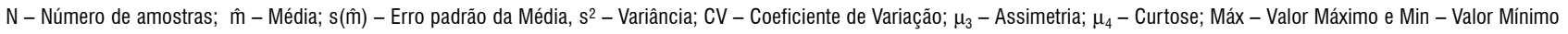

em geral, os índices de assimetria e curtose mostram que, em todos os casos, as variáveis se ajustaram à distribuição de freqüência normal determinada através do teste Shapiro-Wilk.

Apresentam-se, na Tabela 2, os parâmetros dos modelos ajustados aos semivariogramas para as diferentes variáveis estudadas. A variação da razão $\mathrm{k}$ foi de 0,0 a 0,50 , indicando que as variáveis apresentaram um máximo de 50\% de aleatoriedade nos dados de amostragem.

Os semivariogramas e os parâmetros do modelo esférico ajustado aos dados de produtividade, e Gaussiano, para os dados de diâmetro dos frutos, estão representados pela Figura 3A e 3B, respectivamente. Observa-se que o modelo esférico se ajustou aos dados de produtividade (Figura 3A), apresentando dependência espacial (alcance) de $60 \mathrm{~m}$, e uma razão $\mathrm{k}$ de 0,43 , ou seja, os dados de amostragem da produtividade possuem cerca de $43 \%$ de aleatoriedade (Tabela 2); assim, por mais que o modelo esférico esteja indicando dependência espacial dentro da área total estudada (13 ha) existem subáreas com produtividade homogênea.

Tabela 2. Parâmetros dos semivariogramas das variáveis estudadas, ajustados aos modelos

\begin{tabular}{|c|c|c|c|c|c|}
\hline \multirow{2}{*}{ Variáveis } & \multicolumn{3}{|c|}{ Parâmetros } & \multirow{2}{*}{ Modelo* } & \multirow{2}{*}{$\mathbf{k}^{* *}$} \\
\hline & $\mathrm{C}_{0}$ & $C_{1}$ & $a(m)$ & & \\
\hline \multicolumn{6}{|c|}{ Produção } \\
\hline Diâmetro do Fruto & 0,0 & 63,0 & 45,0 & G & 0,0 \\
\hline Produtividade & 100,0 & 135,0 & 60,0 & $\mathrm{E}$ & 0,43 \\
\hline \multicolumn{6}{|c|}{ Solo } \\
\hline pH & 0,002 & 0,041 & 48,0 & E & 0,05 \\
\hline M.0. $\left(\mathrm{g} \mathrm{kg}^{-1}\right)$ & 0,2 & 2,2 & 90,0 & E & 0,08 \\
\hline $\mathrm{P}\left(\mathrm{mg} \mathrm{dm}{ }^{-3}\right)$ & 7,0 & 29,0 & 30,0 & Ex & 0,19 \\
\hline $\mathrm{K}\left(\mathrm{cmol}_{\mathrm{c}} \mathrm{dm}^{-3}\right)$ & 0,0 & 0,002 & 95 & $E$ & 0,0 \\
\hline $\mathrm{Ca}\left(\mathrm{cmol}_{\mathrm{c}} \mathrm{dm}^{-3}\right)$ & 0,005 & 0,105 & 117,0 & $\mathrm{E}$ & 0,50 \\
\hline $\mathrm{Mg}\left(\mathrm{cmol}_{\mathrm{c}} \mathrm{dm}^{-3}\right)$ & 0,002 & 0,008 & 77,0 & $\mathrm{E}$ & 0,20 \\
\hline \multicolumn{6}{|c|}{ Planta } \\
\hline$N\left(\mathrm{~g} \mathrm{~kg}^{-1}\right)$ & 1,30 & 3,0 & 67,0 & E & 0,30 \\
\hline$P\left(g_{k g}^{-1}\right)$ & 0,003 & 0,018 & 95,0 & $\mathrm{E}$ & 0,14 \\
\hline $\mathrm{K}\left(\mathrm{g} \mathrm{kg}^{-1}\right)$ & 0,390 & 0,415 & 76,0 & E & 0,48 \\
\hline $\mathrm{Ca}\left(\mathrm{g} \mathrm{kg}^{-1}\right)$ & 50,0 & 95,0 & 78,0 & E & 0,34 \\
\hline $\operatorname{Mg}\left(\mathrm{g} \mathrm{kg}^{-1}\right)$ & 0,02 & 0,40 & 60,0 & $\mathrm{E}$ & 0,05 \\
\hline
\end{tabular}

Para o diâmetro dos frutos, o modelo que melhor se ajustou aos dados foi o Gaussiano, com dependência espacial de $45 \mathrm{~m}$, apresentando uma razão $\mathrm{k}$ sem aleatoriedade nos dados (Tabela 2), o que se deve ao fato de que a média foi gerada a partir de 10 frutos (repetições), diminuindo o erro nas amostragens.

A Tabela 2 mostra que os modelos foram muito bem ajustados, pois somente o Ca no solo apresentou um parâmetro $\mathrm{k}$ de 0,50, sinal de que, para este macronutriente, cerca de $50 \%$ da variação das amostras são aleatórios; assim, por mais próximas que estejam as unidades amostrais, esta variabilidade estará presente.

Realizou-se, através dos dados dos semivariogramas ajustados, a krigagem ordinária, para estimar as interpolações necessárias para a construção do mapa da distribuição espacial da produtividade e do tamanho do fruto da área. Pelos mapas de isolinhas de produtividade e de tamanho de frutos (Figura 1C e D), pode-se observar as variabilidades espaciais, caracterizando as áreas de maior e menor produtividade e o tamanho de frutos existentes na Quadra DQ12.

O mapa de produtividade (Figura 1C) mostra áreas com maior e menor produção dentro do pomar, variando de 11,25 a 80,8 kg planta-1; as cores mais escuras indicam áreas com elevado potencial de produtividade, que estão bem acima da média.

Os resultados observados neste trabalho estão de acordo com os encontrados por Farias et al. (2003), os quais trabalharam com o mapeamento da produtividade em pomares de cítricos no estado de São Paulo e concluíram que os mapas de produtividade e de tamanho de frutos apresentaram alta variabilidade espacial.

A variabilidade dentro de um pomar de citros também foi observada nos trabalhos de Whitney et al. (1998) e Schueller et al. (1999), que relatam que, sob desigualdade de produção, o aproveitamento dos insumos aplicados uniformemente se torna ineficiente em algumas áreas; além do desperdício desses produtos químico, a contaminação do meio ambiente pode ser incrementada nessas condições.

De acordo com a Figura 1C, a utilização de mapas de produtividade em áreas de citrus representa a informação mais completa para visualizar a variabilidade espacial dos pomares, 


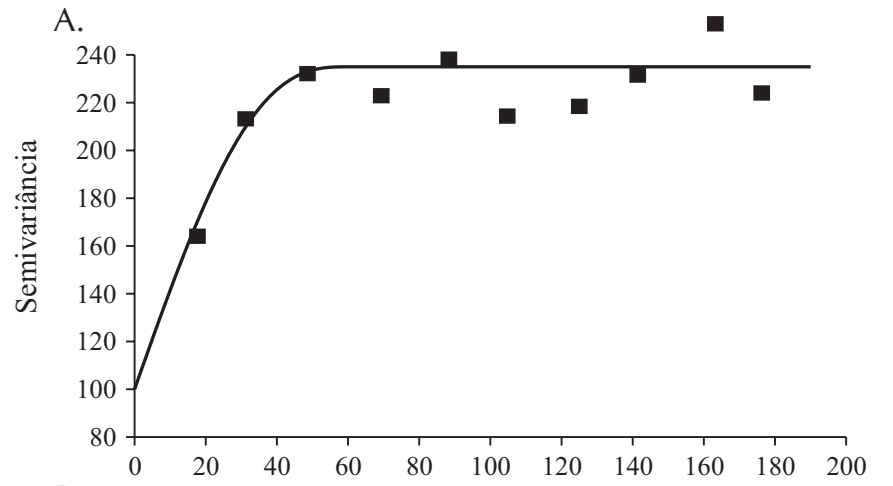

B.
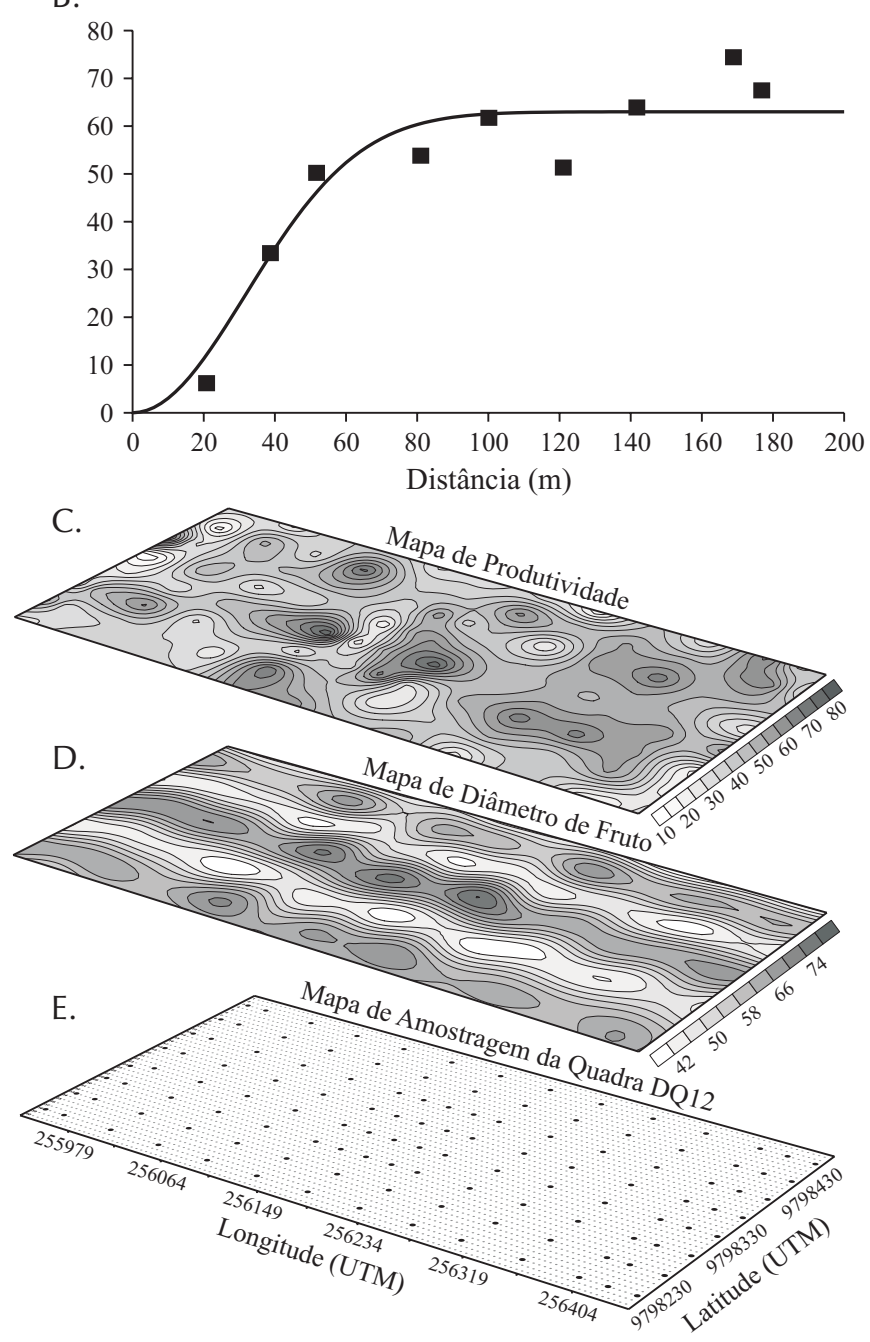

Figura 1. Semivariograma da produtividade (A), tamanho do fruto (B), mapa de produtividade $(\mathrm{C})$, de diâmetro do fruto (D) e mapa de amostragem das plantas georreferenciadas da Quadra DQ12 (E)

materializando a resposta da cultura. A ausência de técnicas e recursos para a geração rotineira de mapas de produtividade em área de citros no Estado do Pará, é uma das grandes dificuldades para a implantação de um sistema de agricultura de precisão, impedindo a adoção da estratégia de gerenciamento integrado de entradas e saídas mas ensejando a mensuração dos efeitos de adoção de taxas variadas de insumos. Esses resultados estão de acordo com os trabalhos de Parise \& Vettorazzi (2005) e Lark \& Stafford (1997); a confecção de mapas de produtividade oferece um indicativo das condições de crescimento e produtividade das plantas, podendo ser utilizado, inclusive, como forma de monitoramento da resposta da cultura às técnicas de manejo utilizadas.

Esta variabilidade na produtividade foi estudada por Annamalai (2004) em citros, na Flórida, o qual concluiu que a aplicação excessiva ou deficiente de insumos, a compactação do solo, a presença de pragas e doenças, dentre outras, é um exemplo de causas artificiais que influenciam a variabilidade espacial nos mapas de produtividade.

Para o diâmetro dos frutos (Figura 1D) o comportamento espacial mostrou tendência de variabilidade, acompanhando as linhas de plantio em razão, provavelmente, do manejo utilizado no pomar, o que influenciou no comportamento espacial do diâmetro de frutos. Com a finalidade de diminuir a incidência de pragas no pomar, utilizou-se o sistema de capinas alternas em que o mato era roçado entrelinha sim, e outra não; na entrelinha não roçada deu-se um aumento na competição por nutrientes o que, sem dúvida, deve ter influenciado o desenvolvimento dos frutos.

As variáveis $\mathrm{pH}$, matéria orgânica, $\mathrm{P}, \mathrm{K}$, Ca e Mg no solo (Figura 2) apresentaram estruturas de dependência espacial, conforme demonstrado pelos modelos ajustados aos semivariogramas, nos quais todas as variáveis se ajustaram ao modelo esférico, com exceção do P, que se ajustou ao modelo exponencial.

O modelo mais adequado para explicar a estrutura da variabilidade espacial do $\mathrm{pH}$ nas amostras de solo, foi o esférico (Figura 1), com um raio de $48 \mathrm{~m}$ (alcance), que representa uma área de $7.238 \mathrm{~m}^{2}$, ou seja, 207 laranjeiras no espaçamento de $5 \times 7 \mathrm{~m}$ (Tabela 2), mostrando uma razão k de 5\% de aleatoriedade nas amostras; este resultado é muito importante para a estimação dos dados no mapa temático. O modelo esférico, representou melhor o comportamento da M.O. no pomar estudado, com uma razão k de $9 \%$ de aleatoriedade nos dados e um raio de $90 \mathrm{~m}$ (alcance) com cerca de $25.000 \mathrm{~m}^{2}$, cobrindo uma área de dependência espacial de aproximadamente 727 plantas (Tabela 2).

Na Tabela 2 se observa que, de maneira geral, os macronutrientes apresentaram dependência espacial nas amostras. O P se ajustou ao modelo exponencial (Figura 2) com um raio de dependência espacial de $30 \mathrm{~m}$ (alcance) e uma razão $\mathrm{k}$ de 19\% de aleatoriedade nos dados; para o K, o modelo mais adequado foi o esférico (Figura 2), muito bem ajustado, indicando um efeito pepita $\left(\mathrm{C}_{0}\right)$ sem aleatoriedade nos dados $(0,0)$. $\mathrm{O}$ raio de dependência espacial foi de 95 m (Tabela 2).

Observa-se, na Figura 2, que o Ca e o Mg se ajustaram ao modelo esférico, com alcances de 117 e $77 \mathrm{~m}$, respectivamente. A aleatoriedade nos dados de amostragem variou entre 20 a 50\% (Tabela 2).

$\mathrm{O}$ modelo que melhor se ajustou aos macronutrientes ( $\mathrm{N}$, P, K, Ca e Mg) da planta, foi o esférico (Figura 3), com um raio de dependência que variou de 60 a 95 m (Tabela 2).

Através dos modelos ajustados aos semivariogramas foi possível realizar a estimativa dos valores amostrados pelo método da krigagem ordinária, para construção dos mapas de produtividade, tamanho do fruto e macronutrientes no solo (Figura 4A) e na planta (Figura 4B). Pelos mapas temáticos de produtividade e de tamanho de fruto sobrepostos aos de 

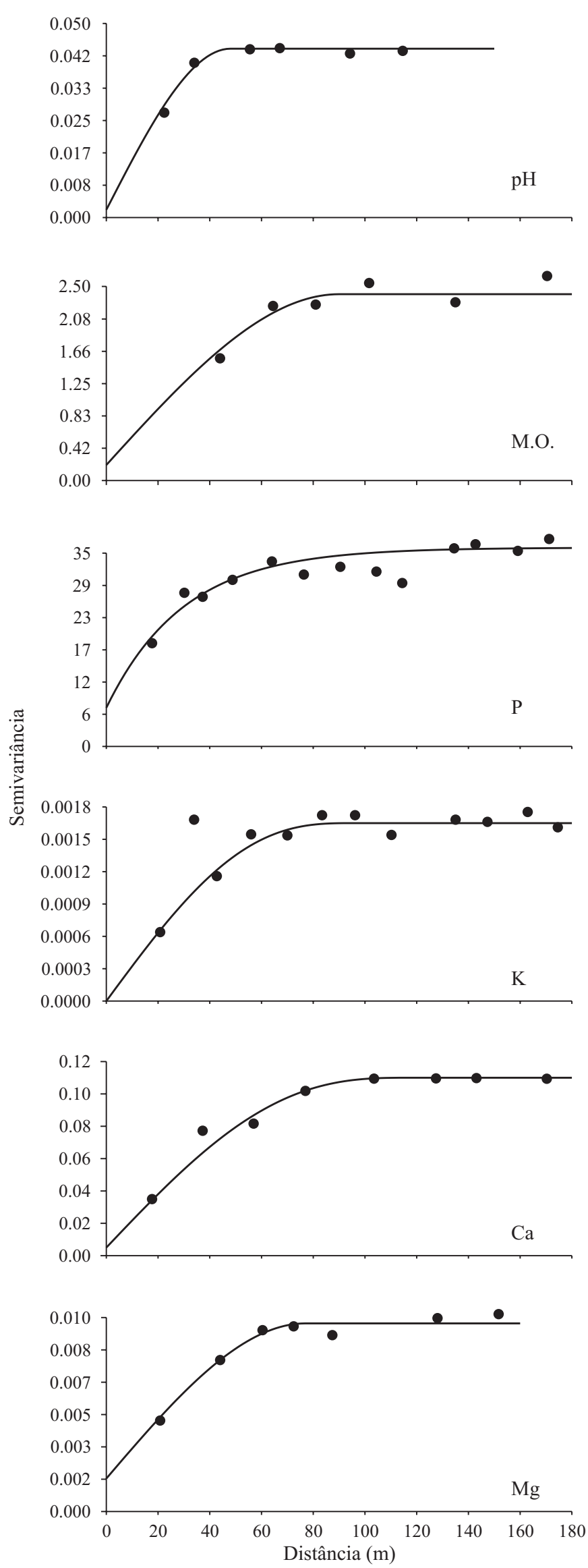

Figura 2. Semivariogramas mostrando os modelos ajustados para $\mathrm{pH}$, matéria orgânica (M.O.), Fósforo (P), Potássio (K), Cálcio (Ca) e Magnésio (Mg) no solo
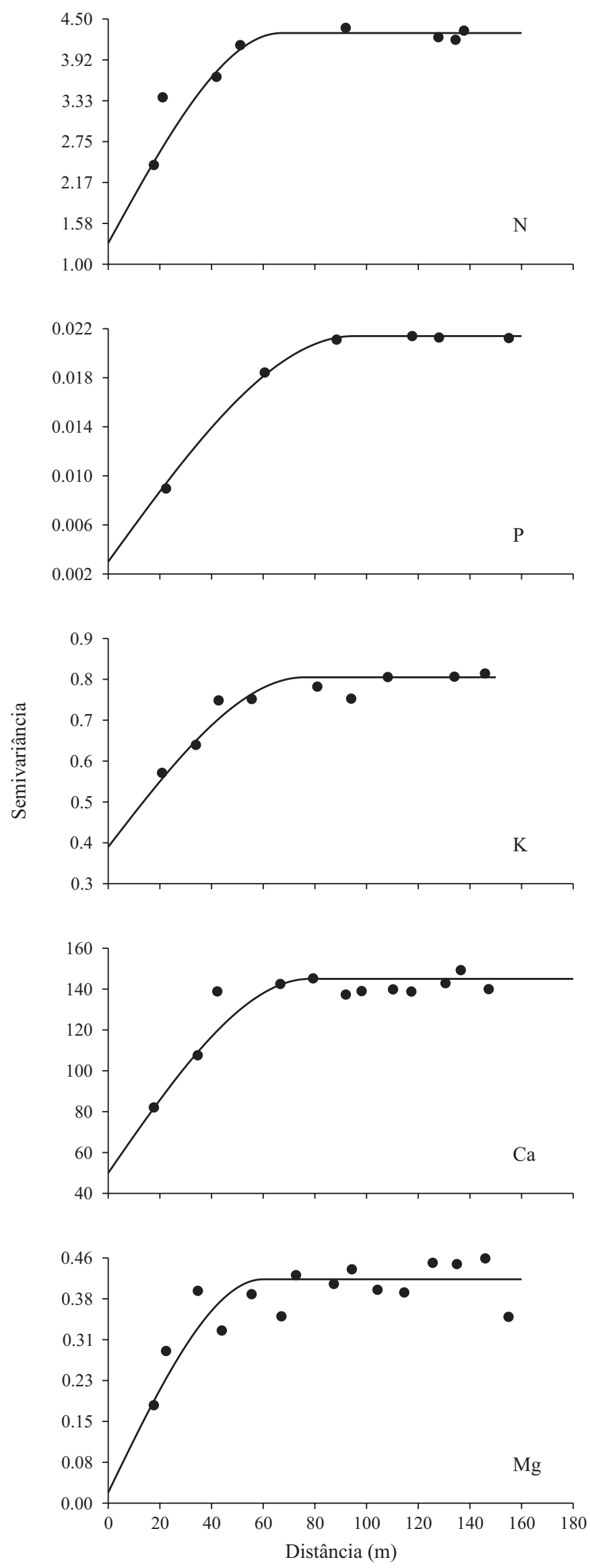

Figura 3. Semivariogramas mostrando os modelos ajustados para Nitrogênio $(\mathrm{N})$, Fósforo (P), Potássio (K), Cálcio (Ca) e Magnésio (Mg) na planta

R. Bras. Eng. Agríc. Ambiental, v.13, n.6, p.708-715, 2009. 
macronutrientes no solo e na planta, pode-se observar as variabilidades espaciais, caracterizando as áreas de risco existentes no pomar.

Pode-se observar, atentando-se para a Figura 4A, que o pH, M.O. e os macronutrientes ( $\mathrm{P}, \mathrm{K}, \mathrm{Ca}$ e $\mathrm{Mg}$ ) no solo apresentam tendência de distribuição espacial seguindo a linha de plantio da cultura, o que pode explicar o comportamento espacial do diâmetro dos frutos, que segue a

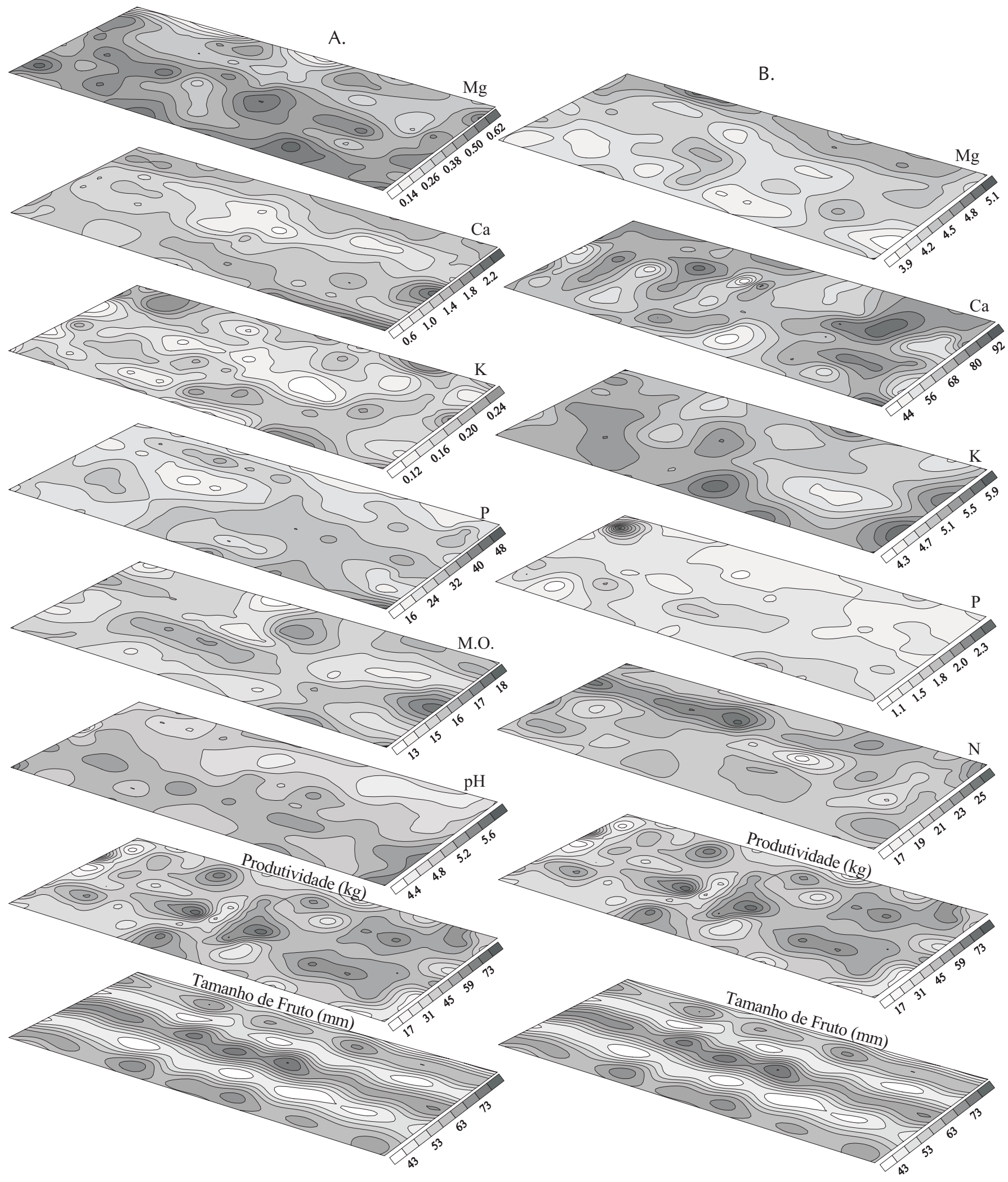

Figura 4. Mapa de variabilidade espacial de tamanho de fruto e produtividade sobreposto aos de $\mathrm{pH}$, matéria orgânica (M.O.), Fósforo (P), Potássio (K), Cálcio (Ca) e magnésio (Mg) no solo (A) e Nitrogênio (N), Fósforo (P), Potássio (K), Cálcio (Ca) e Magnésio (Mg) na planta (B) 
mesma tendência; supõe-se, deste modo, que o manejo das capinas alternadas nas entrelinhas do pomar influenciou diretamente o desenvolvimento e a forte variabilidade no diâmetro dos frutos.

A Figura 4B demonstra que a distribuição espacial dos macronutrientes ( $\mathrm{N}, \mathrm{P}, \mathrm{K}, \mathrm{Ca}$ e $\mathrm{Mg}$ ) na planta segue tendência de distribuição espacial semelhante à variabilidade espacial da produtividade na área devido, provavelmente, à não existência de competição entre as ervas daninhas e a cultura da laranja pelos macronutrientes aplicados através da adubação foliar, prática bastante utilizada no pomar.

As informações geradas através deste trabalho, poderão contribuir no monitoramento e na elevação da produtividade dos pomares de citros na região de Capitão Poço e identificar os prováveis fatores intrínsecos ou extrínsecos responsáveis pela alta variabilidade dos dados, de forma que a utilização de técnicas de manejo diferenciado possa auxiliar na diminuição dos custos e no aumento da produção.

\section{CONCLUSÕES}

1. Os mapas de produtividade e de tamanho de fruto, mostraram alta variabilidade espacial com uma produção variando de 11,25 a $80,8 \mathrm{~kg}$ planta $^{-1}$.

2. O tamanho médio dos frutos apresentou tendência de variabilidade seguindo as linhas de plantio em razão, quiçá, do sistema de alternância de capinas; o tamanho dos frutos variou de 42 a $78 \mathrm{~mm}$.

3. A produtividade não foi influenciada pelo tamanho do fruto e, sim, pelo número de frutos por planta.

4. Todos os macronutrientes analisados no solo e na planta apresentaram dependência espacial.

\section{AgrAdECIMENTOS}

À empresa Cítricos do Pará (CITROPAR), pela disponibilidade da área e pela cooperação de seus funcionários.

\section{LITERATURA CITADA}

Annamalai, P. Citrus yield mapping system using machine vision. Gainesville: University of Florida, 2004, 81p. Tese Doutorado

EMBRAPA - Empresa Brasileira de Pesquisa Agropecuária - Centro Nacional de Pesquisa de Solos: Manual de métodos de análise de solo. 2.ed. Rio de Janeiro: Embrapa-CNPS, 1997. 212p.

FAO. Faostat data 2005. http://faostat.fao.org. 07 Dez. 2007.
Farias, P. R. S.; Barbosa, J. C.; Vieira, S. R.; Sánchez-Vila, X.; Ferraz, L. C. C. B. Geostatistical analysis of the spatial distribution of Rotylenchulus reniformis on cotton cultivated under crop rotation. Russian Journal of Nematology, v.10, n.1, p.1-9, 2002a.

Farias, P. R. S.; Nociti, L. A. S.; Barbosa, J. C.; Perecin, D. Agricultura de precisão: Mapeamento da produtividade em pomares de cítricos usando geoestatística. Revista Brasileira de Fruticultura, v.25, n.2, p.235-241, 2003.

Farias, P. R. S.; Sánchez-Vila, X.; Barbosa, J. C.; Vieira, S. R.; Ferraz, L. C. C. B.; Solis-Delfin, J. Using geostatistical analysis to evaluluate the presence of Rotylenchulus reniformis in cotton crops in Brazil: economic implications. Journal of Nematology, v.34, n.3, p.232-238, 2002b.

Golden Software, INC. (Golden, Estados Unidos). SURFER for windows: realese 8.0, contouring and 3D surface mapping for scientist's engineers user's guide. New York: 2002. 714p.

Guerra, P. A. G. Geoestatística operacional. 1.ed. Brasília: Ministério das Minas e Energia, 1988. 145p.

IBGE - Instituto Brasileiro de Geografia e Estatística. Levantamento sistemático da produção agrícola, 2005. http:// www.ibge.gov.br. 06 Dez. 2007.

Kim, Y. C. Advanced geoestatistics for highly skewed dat. Arizona: Department of Mining and Geological Engineering The University Of Arizona, 1988. 128p.

Lark, R. M.; Stafford, J. V. Classification as a first step in the interpretation of temporal and spatial variation of crop yield. Annals of Applied Biology, v.130, n.1, p.111-121, 1997.

Parise, F. J. de O.; Vettorazzi, C. A. Análise de dados de produção em um pomar jovem de laranjeiras hamlin: Classificação de dados espaço-temporais. Revista Brasileira de Fruticultura, v.27, n.1, p.48-51, 2005.

Schueller, J. K.; Whitney, J. D.; Wheaton, T. A.; Miller, W. M.; Turner, A. E. Low-cost automatic yield mapping in hand-harvested citrus. Computers and Electronics in Agriculture, v.23, n.3, p.145-153, 1999.

Silva, F. C. Manual de análises químicas de solos, plantas e fertilizantes. 1.ed. Brasília: EMBRAPA, 1999. 369p.

Souza, L. S.; Cogo, N. ; Vieira, S. R. Variabilidade de propriedades físicas e químicas do solo em um pomar cítrico. Revista Brasileira de Ciência do Solo, v.21, n.3, p.367-372, 1997.

Vieira, S. R.; Hatfield, J. L.; Nielsen, D. R.; Biggar, J. W. Geoestatistical theory and application to variability of some agronomical properties. Hilgardia, v.51, n.3, p.1-75, 1983.

Whitney, J. D.; Wheaton, T. A.; Miller, W. M.; Salyani, M.; Schueller, J. K. Precision farming applications in Florida Citrus. Proceedings of Florida State Horticultural Society, v.111, p.148-150, 1998.

Wilson, L. T.; Room, P. M. Clumping patterns of fruit and arthropods in cotton with implications for binomial sampling. Environmental Entomology, v.12, n.2 p.50-58, 1983. 